\title{
An Efficient Hairy Root System for Validation of Plant Transformation Vector and CRISPR/Cas Construct Activities in Cucumber (Cucumis sativus L.)
}

\section{OPEN ACCESS}

Edited by:

Kejian Wang,

China National Rice Research

Institute, Chinese Academy of Agricultural Sciences (CAAS), China

Reviewed by: Jitesh Kumar, University of Minnesota Twin Cities, United States

Yoshihiko Nanasato, Forestry and Forest Products

Research Institute, Japan

${ }^{*}$ Correspondence: Ha Hoang Chu chuhoangha@ibt.ac.vn Phat Tien Do dtphat@ibt.ac.vn

tThese authors have contributed equally to this work and share first authorship

Specialty section: This article was submitted to Plant Biotechnology, a section of the journal Frontiers in Plant Science

Received: 03 September 2021 Accepted: 27 December 2021 Published: 11 February 2022

Citation: Nguyen DV, Hoang TT-H, Le NT, Tran HT, Nguyen CX, Moon Y-H, Chu HH and Do PT (2022) An Efficient Hairy Root System for Validation of Plant Transformation Vector and CRISPR/Cas Construct Activities in Cucumber (Cucumis

Front. Plant Sci. 12:770062. doi: 10.3389/fpls.2021.770062
Doai Van Nguyen ${ }^{1,2 \dagger}$, Trang Thi-Huyen Hoang ${ }^{1 \dagger}$, Ngoc Thu Le ${ }^{1}$, Huyen Thi Tran ${ }^{1}$, Cuong Xuan Nguyen ${ }^{3}$, Yong-Hwan Moon ${ }^{2,4}$, Ha Hoang Chu ${ }^{1,5 *}$ and Phat Tien Do ${ }^{1,5 *}$

'Laboratory of Plant Cell Biotechnology, Institute of Biotechnology, Vietnam Academy of Science and Technology, Hanoi, Vietnam, ${ }^{2}$ Department of Integrated Biological Science, Pusan National University, Busan, South Korea, ${ }^{3}$ Division of Plant Sciences, University of Missouri, Columbia, MO, United States, ${ }^{4}$ Department of Molecular Biology, Pusan National University, Busan, South Korea, ${ }^{5}$ Graduate University of Science and Technology, Vietnam Academy of Science and Technology, Hanoi, Vietnam

Hairy root induction system has been applied in various plant species as an effective method to study gene expression and function due to its fast-growing and high genetic stability. Recently, these systems have shown to be an effective tool to evaluate activities of CRISPR/ Cas9 systems for genome editing. In this study, Rhizobium rhizogenes mediated hairy root induction was optimized to provide an effective tool for validation of plant transformation vector, CRISPR/Cas9 construct activities as well as selection of targeted gRNAs for gene editing in cucumber (Cucumis sativus L.). Under the optimized conditions including $\mathrm{OD}_{650}$ at 0.4 for infection and 5 days of co-cultivation, the highest hairy root induction frequency reached $100 \%$ for the cucumber variety Choka F1. This procedure was successfully utilized to overexpress a reporter gene (gus) and induce mutations in two Lotus japonicus ROOTHAIRLESS1 homolog genes CsbHLH66 and CsbHLH82 using CRISPR/Cas9 system. For induced mutation, about $78 \%$ of transgenic hairy roots exhibited mutant phenotypes including sparse root hair and root hair-less. The targeted mutations were obtained in individual CsbHLH66, CsbHLH82, or both CsbHLH66 and CsbHLH82 genes by heteroduplex analysis and sequencing. The hairy root transformation system established in this study is sufficient and potential for further research in genome editing of cucumber as well as other cucumis plants.

Keywords: cucumber, hairy root, Rhizobium rhizogenes, CRISPR/Cas9, root hair-less, CsbHLH66, CsbHLH82, ROOTHAIRLESS1

\section{INTRODUCTION}

Originating in the Indian subcontinent, cucumber (Cucumis sativus L.) has become one of the top consumed vegetables widely grown in the world (Paris et al., 2012; Taha et al., 2020). However, cucumber cultivation currently has suffered from biotic and abiotic stresses (Liu et al., 2013; Chojak-Koźniewska et al., 2018; Fan et al., 2020; Taha et al., 2020). There 
are various approaches used to improve cucumber production such as optimizing cultivation conditions (Taha et al., 2020), plant grafting (Elsheery et al., 2020), and breeding. Of which, modem breeding with the help of genetic engineering has been known as the most effective method because genetic variability of cucumber varieties is low 3-8\% (Chee, 2001; Plader et al., 2007). The genome editing boosted by CRISPR/ Cas systems has been widely applied on various crops as a powerful tool to improve plant traits and gene function studies (Zhang et al., 2021). In cucumber, there have been several reports of using CRISPR/Cas9 for targeted gene knockout (Chandrasekaran et al., 2016; Hu et al., 2017). However, applications of genome editing in cucumber have been challenged by less effective transformation procedures that are time consuming and labor extensive processes ( $\mathrm{Hu}$ et al., 2017). Therefore, the improvement of procedures and the development of supporting tools are necessary to accelerate genetic engineering in cucumber.

Rhizobium rhizogenes-mediated hairy root cultures have been established and developed in different plant species for biomass, recombinant protein production, gene function studies, and secondary metabolite researches (Gutierrez-Valdes et al., 2020). In addition, hairy roots also could be used as materials for generating whole plants in certain reports (Trulson et al., 1986; Bueno dos Reis et al., 2007; Butler et al., 2020; Zhang et al., 2020). Recently, the hairy root transformation has been widely utilized to validate and optimize induced mutagenesis by the CRISPR/Cas9 system (Michno et al., 2015; Du et al., 2016; Jacobs and Martin, 2016; Li et al., 2019; Le et al., 2020). Both in vitro and in vivo cucumber hairy root cultures have been established for different research purposes (Shi and Lindemann, 2006; Anuar et al., 2011; Fan et al., 2020). However, the application of cucumber hairy root transformation for genome editing using CRISPR/Cas systems has not been reported yet. Moreover, it has been reported that hairy root induction frequency is affected by cucumber varieties, bacterial strains, and transformation methods (JingLong et al., 2017; Fan et al., 2020).

In this study, an efficient procedure for in vitro cucumber hairy root induction and transformation was established for a local cucumber cultivar. This system was also applied for the expression of a reporter gene $(g u s)$ as well as validation of CRISPR/Cas construct activity. The targeted mutations including heterozygous, homozygous, and biallelic indels in two targeted genes CsbHLH66 and CsbHLH82 was obtained at primary and propagated transgenic hairy roots. These results provide an effective tool for gene expression, gene function, and genome editing studies in cucumber.

\section{MATERIALS AND METHODS}

\section{Plant Material}

Seeds of a local cucumber (Cucumis sativus L.) cultivar Choka F1 were surface sterilized in 15\% commercial bleach (bleaching solution containing 5\% sodium hypochlorite, Duc Giang, $\mathrm{VN}$ ) for $15 \mathrm{~min}$, then washed 4-5 times in sterile water
(Hoang et al., 2021). The sterilized seeds were soaked in autoclaved water for $3 \mathrm{~h}$ at $26^{\circ} \mathrm{C}$. After removing seed coats, unwounded seeds were placed on MS30 medium (Table 1) for 2 days in the dark.

\section{CRISPR/Cas9 Vector Construction for Hairy Root Assay}

The CsbHLH66 and CsbHLH82 and other related protein sequences were discovered by the BlastP (NCBI) using the Lotus japonicus ROOTHAIRLESS1 (LjRHL1) protein sequence (Karas et al., 2009) as a reference. To induce targeted mutations in CsbHLH66 and CsbHLH82 genes, gRNAs were generated by the CCTop program (Stemmer et al., 2015). The CRISPR/ Cas9 plant transformation vectors were constructed based on the pKSE401 vector (addgene plasmid \# 62202), a gift from Qi-Jun Chen laboratory (Xing et al., 2014), in which, the neomycin phosphotransferase II (ntpII) gene was replaced by the basta resistance (bar) gene. The coding sequence of bar gene was amplified from the binary vector pZY102 (Zeng et al., 2004) using primer pairs: NcoI-bar-F and RsrII-bar-R (Supplementary Table S1). The PCR amplifications were conducted using the Phusion High-Fidelity DNA Polymerase kit (Cat. No. F553L - Thermo Scientific) following the manufacturer's instructions. After purification, the PCR products were inserted into pKSE401 at NcoI and RsrII sites. For gRNA insertion, BsaI-CsbHLH66 gRNA-gRNA scaffold-U6 terminator$B s a \mathrm{I}$ and $B s a \mathrm{I}-U 6$ promoter-CsbHLH82-gRNA-BsaI fragments were generated from the pKSE401 template by PCR using the listed primers (Supplementary Table S1). These two fragments were assembled into the destination vector pKSE401 using BsaI enzyme sites. The final constructed vector was confirmed by sequencing and named pKSE401-CsbHLH82-66. The pKSE401CsbHLH82-66 and pZY102 binary vectors were mobilized into $R$. rhizogenes strain $\mathrm{K} 599$ and used for cucumber hairy root transformation.

\section{Rhizobium rhizogenes-Mediated Transformation and Hairy Root Induction}

Rhizobium rhizogenes strains including ATCC 15834 (pRi15834) and K599 (pRi2659) wild-type or carrying binary vectors were used for bacterial mediated transformation. Briefly, single colonies

TABLE 1 | The list of media used in cucumber hairy root transformation.

\begin{tabular}{ll}
\hline Medium & Composition or references \\
\hline MS & Murashige and Skoog, 1962 \\
MS30 & MS, $30 \mathrm{~g} / \mathrm{L}$ sucrose and $7.5 \mathrm{~g} / \mathrm{L}$ agar; \\
& $\mathrm{pH} 5.8$ \\
Re-suspension & $0.5 \mathrm{MS}, 30 \mathrm{~g} / \mathrm{L}$ sucrose and $200 \mu \mathrm{M} / \mathrm{L}$ \\
& $\mathrm{AS} ; \mathrm{pH} 5.8$ \\
Co-cultivation & $\mathrm{MS} 30,200 \mu \mathrm{M} \mathrm{AS}, \mathrm{pH} 5.8$ \\
Hairy root culture & $\mathrm{MS} 30,300 \mathrm{mg} / \mathrm{L}$ cefotaxime; $\mathrm{pH} 5.8$ \\
Hairy root selection & Hairy root culture medium added \\
& selective agents
\end{tabular}

AS, antibiotics and selective agents were added to medium after autoclaving. 
were cultured in $5 \mathrm{ml}$ liquid yeast extract peptone (YEP) medium supplemented with appropriate antibiotics $(25 \mathrm{mg} / \mathrm{L}$ rifampicin for the $R$. rhizogenes strain K599; no antibiotic for the $R$. rhizogenes strain ATCC 15834; $25 \mathrm{mg} / \mathrm{L}$ rifampicin plus $50 \mathrm{mg} / \mathrm{L}$ kanamycin the $R$. rhizogenes strain K599 harboring pKSE401-CsbHLH82-66 vector; $25 \mathrm{mg} / \mathrm{L}$ rifampicin plus $100 \mathrm{mg} / \mathrm{L}$ spectinomycin for the $R$. rhizogenes strain K599 haboring pZY102 vector). The culture was shaken at $28^{\circ} \mathrm{C}$ and $200 \mathrm{rpm}$ for $4-6 \mathrm{~h}$, transferred to $100 \mathrm{ml}$ of fresh medium and cultured overnight until $\mathrm{OD}_{650}$ reached $0.8-1.0$. On the next day, the cells were harvested by centrifugation at $3,000 \mathrm{rpm}$ for $15 \mathrm{~min}$ and re-suspended in the resuspension medium (Table 1) to get the $\mathrm{OD}_{650}$ of 0.4 .

The cotyledons from 2-day-old seedlings were collected and used as explants for infection. The distal parts and proximal petioles were excised and removed. Then, the proximal parts of the cotyledons were cut in half along the midrib to get small pieces (Figures 1A,B). Cotyledon segments were kept on wet filter papers during the cutting time to avoid water loss. About 60-70 explants were soaked in $30 \mathrm{ml}$ bacterial suspension for $30 \mathrm{~min}$ at room temperature (Figure 1C). Then, explants were removed from the extra liquid and placed on the co-cultivation medium (Table 1) covered by a filter paper (Whatman \# 1001-090) on top. The culture plates were kept at $25 \pm 2{ }^{\circ} \mathrm{C}$ in the dark for 5 days. Next, explants were washed by autoclaved water, blotted by sterile filter papers, and placed on the hairy root culture medium (Table 1) for 15-20 days. Root tip fragments $(2 \mathrm{~cm})$ were cut and transferred to fresh medium for elongation and development. Transgenic hairy roots were obtained on the hairy root selection medium (Table 1) for 10-15 days (Figure 1E). Cucumber hairy root induction and selection were conducted at $25 \pm 2{ }^{\circ} \mathrm{C}$ under a light density of 2,000 lux with a photoperiod of $16 / 8 \mathrm{~h}$.

\section{GUS Histochemical Analysis}

The pZY102 transformed hairy roots survived on the selection medium were collected for GUS histochemical analysis using 5-bromo-4-chloro-3-indolyl glucuronide buffer at $37^{\circ} \mathrm{C}$ for $16 \mathrm{~h}$ (Jefferson et al., 1987). GUS expression in cucumber hairy roots was observed and captured by a regular camera.

\section{Identification and Characterization of Induced Mutant Hairy Roots}

Root hair phenotypes were analyzed using the method described by Karas et al. $(2005,2009)$, in which root hairs were observed under ZEISS Stemi 305 stereo microscope and captured with AxioCam ERc $5 \mathrm{~s}$ digital camera. Genomic DNA was extracted from hairy roots using the CTAB method (Doyle and Doyle, 1987). Hairy roots with induced mutations in CsbHLH genes were detected by heteroduplex analysis on polyacrylamide gel electrophoresis (PAGE; Chen et al., 2012) with some modifications. Briefly, the spanning target regions on the CsbHLH genes were amplified using flanking specific primers (Supplementary Table S1). The PCR amplifications were conducted using DreamTaq Green PCR Master Mix (Cat. No. K1081 - Thermo Scientific) with the following conditions: initial denaturation at $94^{\circ} \mathrm{C}$ for $4 \mathrm{~min} ; 30$ cycles at $94^{\circ} \mathrm{C}$ for $20 \mathrm{~s}, 55^{\circ} \mathrm{C}$ for $20 \mathrm{~s}$ and $72^{\circ} \mathrm{C}$ for $30 \mathrm{~s}$, and a final extension
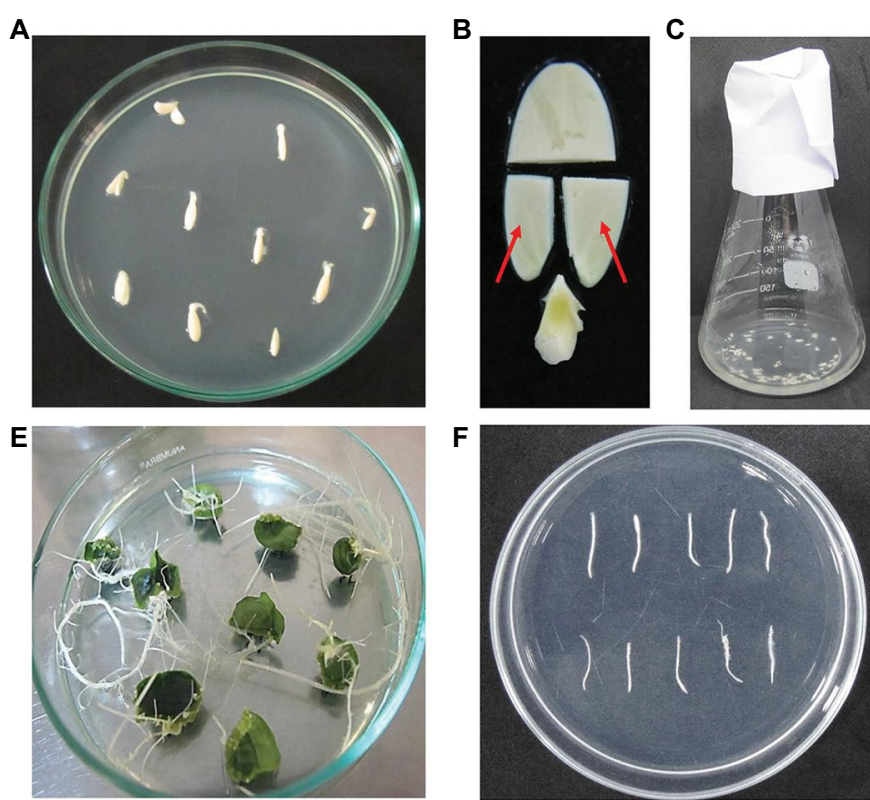
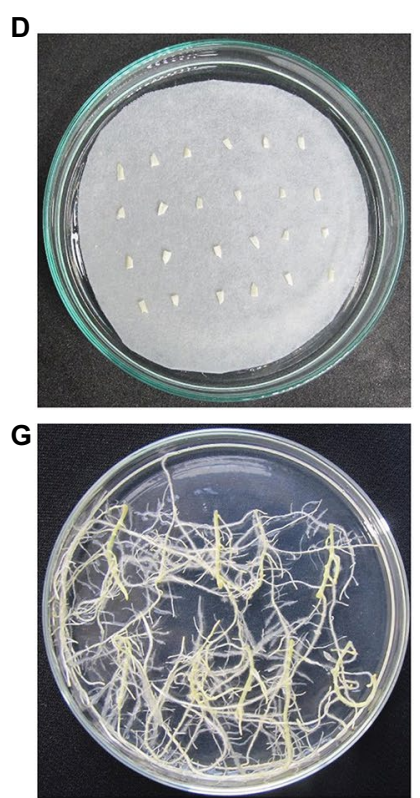

FIGURE 1 | Main steps of cucumber hairy root induction. (A) The 2-day-old seedlings; (B) cotyledon explants preparation. The red arrows indicate the cotyledon fragments used for bacterial infection; (C) cotyledon fragments were soaked in bacterial suspension; (D) the infected explants on co-cultivation medium; (E) hairy root formation at 20 days after co-cultivation; (F) hairy root fragments were transferred to a new medium; (G) hairy roots developed on hairy root culture medium. 
at $72^{\circ} \mathrm{C}$ for $5 \mathrm{~min}$. The mixture containing equal amplicons from wild type (WT) and transgenic hairy roots was denatured at $95^{\circ} \mathrm{C}$ for $10 \mathrm{~min}$ and slowly cooled down to room temperature to form heteroduplexes. The heteroduplexes were detected by PAGE using $12 \%$ polyacrylamide gel. The PCR amplicons were further purified and ligated to the pJET1.2 cloning vector (Thermo FisherScientific, United States) for sequencing. The sequencing was performed on the ABI3500XL system (Applied Biosystems). CsbHLH mutant sequences were compared to wild-type sequences using BioEdit 7.2.

\section{Statistical Analysis}

All statistical analyses were performed using SPSS software version 24.1. The significant analysis (value of $p<0.05$ ) between experiments was performed by Student's $t$-test or one-way ANOVA followed by post-hoc Duncan's new multiple range tests.

\section{RESULTS}

\section{Optimization of Cucumber Hairy Root Induction Procedure}

In this study, cotyledon pieces from 2-day-old seedlings of ChokaF1 cultivar were first inoculated with $R$. rhizogenes K599 strain to evaluate the effects of co-cultivation duration on induced hairy root efficacy. After 3, 5, and 7 days of co-culture, explants were transferred onto hairy root induction medium. There were $21.1 \pm 1.6 \%$ explants of the 5 -day co-cultivation treatment that showed hairy root formation at 5 days on induction medium, and it dramatically reached $100 \%$ at 20 days after co-cultivation (Figure 2A). While the highest rate of explants in 3- and 7-day co-cultivation producing hairy roots after 20 days were $84.4 \pm 1.6 \%$ and $65.6 \pm 3.14 \%$, respectively, and no more explants produced hairy roots if having the culture period prolonged. Bacterial overgrowth was also observed in the 7-day co-cultivation treatment (data not shown). Therefore, 5 -day co-cultivation was sufficient for inducing hairy roots from the local cucumber ChokaF1 and further used for all cucumber hairy transformation experiments.

The effects of $R$. rhizogenes strain (ATCC 15834), a widely used $R$. rhizogenes strain to induce hairy roots in various plant species including cucumber (Shi et al., 2006; Shi and Lindemann, 2006), were also evaluated on the local cucumber cultivar in comparison to the K599 strain (Figure 2B). In this experiment, explants inoculated with ATCC 15834 strain produced hairy roots earlier than those infected with the K599 strain. About $33.3 \pm 11.9 \%$ explants induced hairy roots $(2-3 \mathrm{~cm})$ after 1 week on hairy root induction medium, whereas no hairy root was observed from explants infected with K599. After 10 days, the rate of explants producing hairy roots was $34.4 \pm 4.2 \%$ for K599, while it was double $(61.1 \pm 18.1 \%)$ for the ATCC 15834 strain. The number of ATCC 15834 inoculated explants generating hairy root was continuously higher than K599 treated ones after 15 days. However, after 20 days the highest hairy root induction frequency from ATCC 15834 infected explants was lower $(83.3 \pm 16.6 \%)$ than the ones from K599 (100\%). In addition, the formation of calli were observed from wounding sites as well as whole explants which were inoculated with the ATCC 15834 strain. Whereas very few calli formed from K599 inoculation (Supplementary Figure S1).

From all the above results, an optimized procedure for cucumber in vitro hairy root induction was established, in which cotyledons of 2-day seedlings were infected with $R$. rhizogenes $\mathrm{K} 599$ and co-cultivated for 5 days. The total time to conduct the procedure is less than 1 month and the hairy root induction rate could reach up to $100 \%$. Details of the procedure are presented in Figures 1, 3.

\section{Utilizing Cucumber Hairy Root System for Validating Plant Transformation Vector Activities and Transgene Expression}

Utilizing the developed hairy root system, the expression of transgenes was conveniently assessed. K599 strain harboring binary vector pZY102 carrying a reporter gene (gus) and
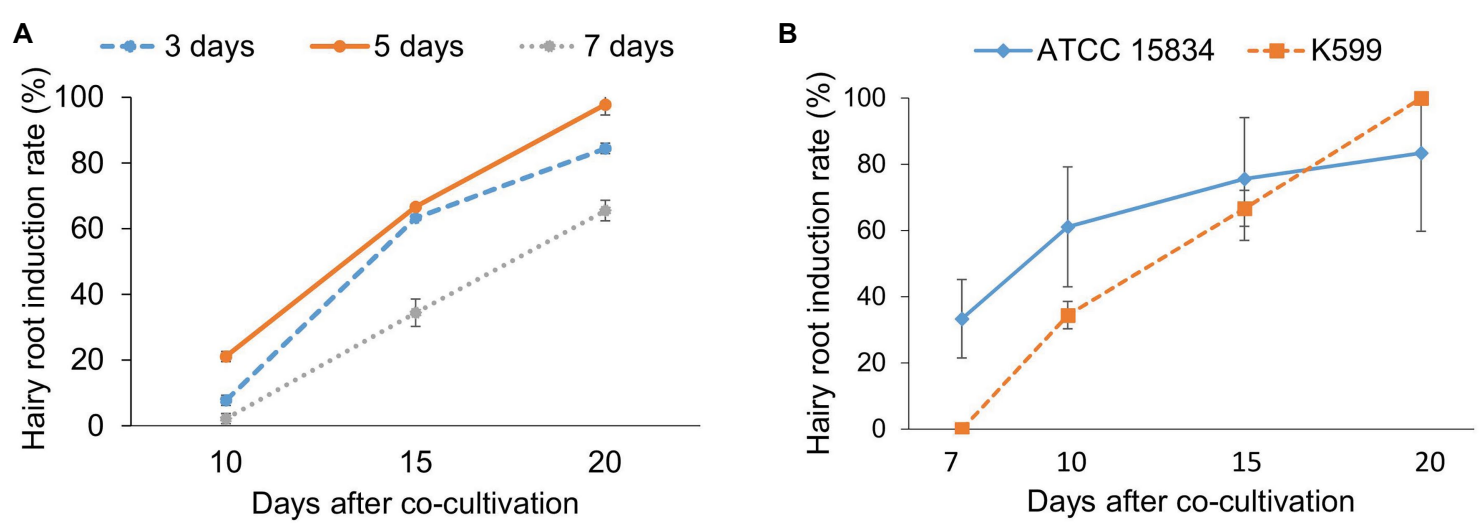

FIGURE 2 | The effects of co-cultivation duration and bacterial strains on cucumber hairy root induction. (A) Co-cultivation time and hairy root induction. (B) Rhizobium rhizogenes strains affect hairy root induction. Hairy root induction was observed and counted at different time points. Data are presented as mean \pm SD of three replicates. About 30 explants were used for each treatment. 


\section{Cucumber mature seeds}

Sterilization

(15\% commercial bleach for 15 minus, washing by sterile water for several times).

Seed germination

(Remove the seed coats, Inoculate in MS30 solid medium)

$\downarrow$ (2 days $)$

Explant preparation

(Remove shoot tips, cut cotyledons into $3 \times 3 \mathrm{~mm}$ pieces).

\section{R. rhizogenes $\mathrm{K} 599$ primary culture}

(Grow single colonies on $5 \mathrm{ml}$ YEP medium + antibiotics at $28^{\circ} \mathrm{C}$ and $250 \mathrm{rpm}$ shaking for $4-6 \mathrm{~h}$ )

\section{R. rhizogenes K599 secondary culture}

(Transfer bacteria to $100 \mathrm{ml}$ same medium, grow in similar condition overnight to get $\mathrm{OD}_{650}$ of $0.8-1$ )

\section{Bacterial cell collection}

(Centrifuge at $3500 \mathrm{rpm}$ for $15 \mathrm{~min}$ at $25^{\circ} \mathrm{C}$ )

Bacterial suspension

(Re-suspend bacterial cell pellets in re-suspension medium to get $\mathrm{OD}_{650}=0.4$ )

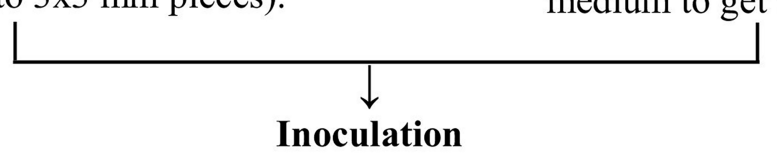

(Soak cotyledon pieces in bacterial suspension)

\section{$\downarrow$ (30 minutes) \\ Co-cultivation}

(Culture on co-cultivation medium in dark at $25^{\circ} \mathrm{C}$ )

$$
\downarrow \text { (5 days) }
$$

\section{Hairy root induction}

(Wash cotyledon pieces by sterile water and transfer to hairy root culture medium )

$$
\downarrow \text { (15 - } 20 \text { days })
$$

\section{Selection}

Transfer hairy root tips $(2 \mathrm{~cm})$ to hairy root culture medium or selection medium (for transgenic hairy root selection)

$$
\downarrow \text { (10 - } 15 \text { days })
$$

\section{Hairy root collection}

FIGURE 3 | The procedure for cucumber hairy root induction using cotyledons.

selective gene (bar) was transferred into cucumber cotyledon pieces. The induced hairy roots $(2 \mathrm{~cm})$ were removed from explants and cultured on the selection medium containing herbicide phosphinothricin (PPT) concentrations ranging from 0.5 to $5 \mathrm{mg} / \mathrm{L}$. The herbicide-resistant hairy roots showed normal morphology and development, while non-resistant hairy roots were completely inhibited and turned yellow without elongation (Figure 4A). The analysis of gus gene expression in putative transgenic hairy roots (herbicide resistant ones) after 10 days on selection medium indicated that not all of them were positive for GUS staining (Figures 4B,C; Supplementary Figure S2). The number of selection-escape hairy roots was varied and depended on
PPT concentration. On the medium with 3 or $5 \mathrm{mg} / \mathrm{L}$ PPT, $6.7 \pm 1.6 \%$ of survived hairy roots escaped. This number raised up to $46 \pm 7.2 \%$ and $48.7 \pm 7.6 \%$ in the medium containing 1.0 and $0.5 \mathrm{mg} / \mathrm{L}$ PPT, respectively. On the 15 th day in selection medium of $3 \mathrm{mg} / \mathrm{L}$ PPT, the number of GUS positive/negative hairy roots was not significantly different from hairy roots on the 10th day (Figure 4C). This result indicates that transgenic hairy roots could be effectively obtained in $3 \mathrm{mg} / \mathrm{L}$ PPT medium in 10 days with the lowest rate of escaping from the selection. Therefore, the activity of the binary vector and the expression of transgenes could be effectively analyzed using the cucumber in vitro hairy root system for 10-15 days. 
A

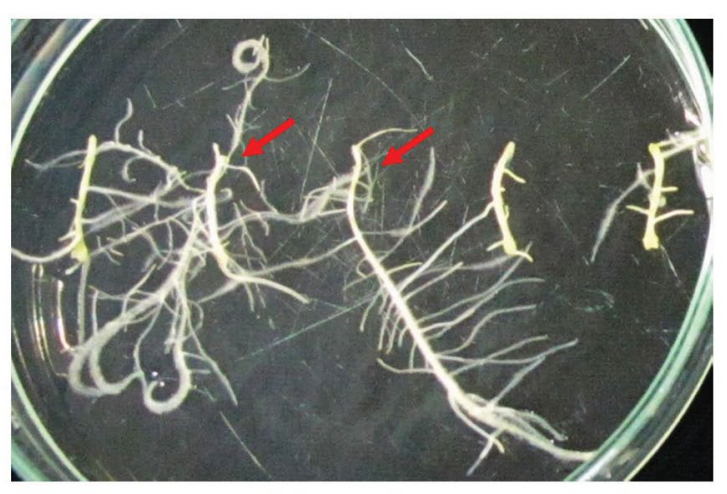

B

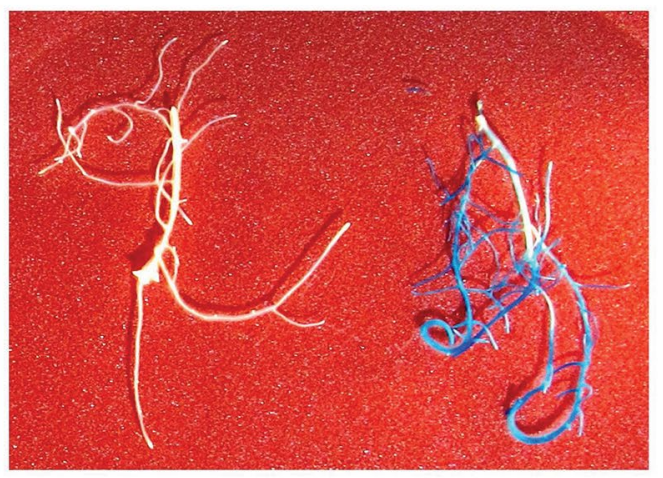

C

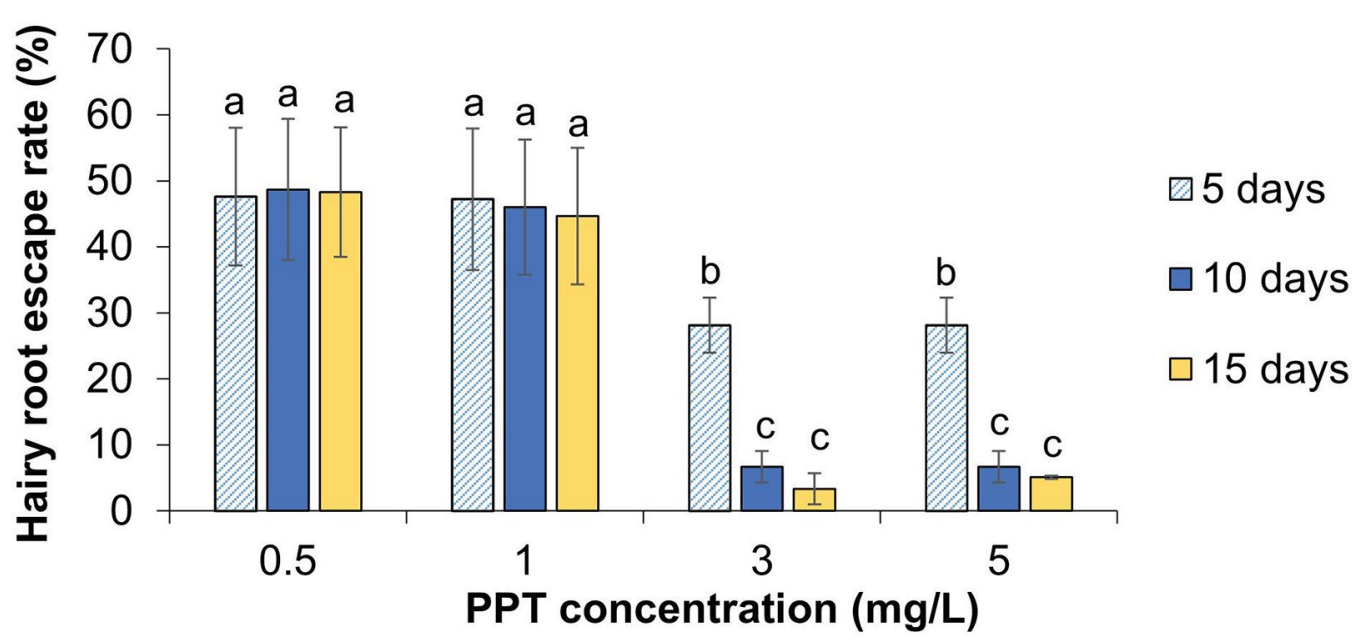

FIGURE 4 | The transgenic hairy root selection and histochemical analysis. (A) Hairy roots at 10 days on selection medium supplemented with $3 \mathrm{mg} / \mathrm{L}$ PPT. The red arrows indicate the PPT resistant hairy roots. (B) GUS histochemical analysis of non-transgenic (left) and transgenic hairy roots (right). (C) Effect of PPT concentrations on the selection efficiency. The escaped hairy roots are referred to the PPT resistant hairy roots without GUS staining. Data are presented as mean \pm SD of three replicates. Different letters indicate significant differences based on one-way ANOVA analysis with Duncan's test $(p<0.05, n=30)$.

\section{Identification of Targeted Genes for CRISPR/Cas9 Induced Mutagenesis of Cucumber Hairy Root}

In addition to testing the transgene expression, the developed hairy root induction system in this study was applied for genome editing studies utilizing the CRISPR/Cas9 system. Four candidate sequences, namely, CsbHLH82, CsBHLH66, CsUNE12, CsBHLH84, were identified when the LjRHL1 protein sequence (from Lotus japonicus, Karas et al., 2009) was used as a reference for searching on cucumber database (NCBI: ASM407v2) by protein BLAST tool. The phylogenetic tree was constructed using the four candidate sequences, the LjRHL1 sequence, and 3 ortholog sequences from Arabidopsis thaliana by the maximum likelihood method and JTT matrix-based model in MEGA X (Kumar et al., 2018). The result indicated that CsbHLH82 and CsbHLH66 were highly close to LjRHL1 (Figure 5A). Moreover, the bHLH domain sequences of the CsbHLH82 and CsbHLH66 were identical to the one of the LjRHL1 (Figure 5B). Therefore, both CsbHLH82 and CsbHLH66 were selected as the targets for mutagenesis in the cucumber hairy roots.

\section{Inducing Targeted Mutations in Cucumber Hairy Roots by CRISRP/Cas9 System}

The DNA sequences of both CsbHLH82 and CsbHLH66 genes were reconfirmed in the local cucumber variety for developing a CRISPR/Cas9 vector. Two gRNAs targeting the first exon of CsbHLH66 and CsbHLH82 were identified using the CCTop tool (Figure 6A) and inserted in the CRISPR/Cas9 expression binary vector (Figure 6B) with the purpose to induce individual and simultaneous targeted mutations of the two tested genes.

Targeted mutations of CsbHLH82 and CsbHLH66 were induced in hairy roots using the K599 strain that harbored the CRISPR/Cas9 vector. Each induced hairy root considered as an independent line was transferred to the selection medium. Phenotyping based on more than 80 hairy root lines indicated that there were three morphological groups (Figure 6C; Supplementary Figure S3) including (1) abundant root hairs like wild-type hairy roots $(22.04 \%)$, (2) sparse and short root hairs $(52.54 \%)$, and (3) root hair-less (25.42\%). 
A
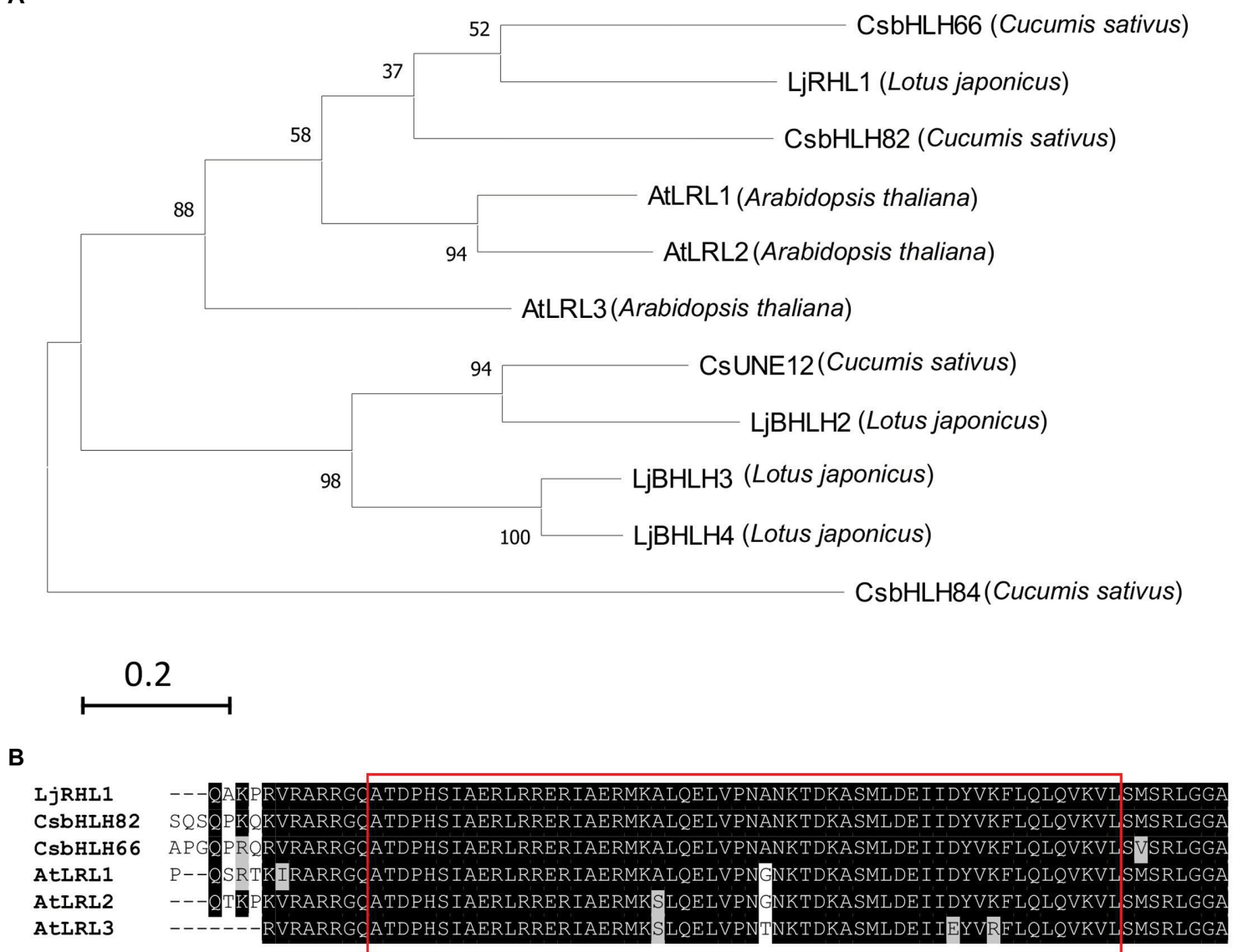

bHLH domain

FIGURE 5 | The phylogenetic tree and sequence analysis of the LjRHL1 homologs. (A) Molecular phylogenetic tree of LjRHL1 protein sequence and its homologs were conducted by MEGA X using the maximum likelihood method and JT matrix-based model. Numbers at nodes indicate the percentage bootstrap scores from 1,000 replicates. The scale bar represents 0.2 estimated number of amino acid substitution events per site. (B) Sequence alignment bHLH domain (in red box) of LjRHL1 in Lotus japonicus and its homologs in cucumber and Arabidopsis.

Heteroduplex analysis of 13 randomly selected hairy root lines from group 2 and 3 (Figures 7A,B) showed that all tested lines contained mutations on CsbHLH82 upon the shifted DNA band observation on the PAGE gel as compared to the wild type hairy roots (Figure 7A). In the case of CsbHLH66, only 4 hairy root lines $(3,6,10,30)$ exhibited induced mutations (Figure 7B). Importantly, these 4 lines all belonged to group 3 having the root hair-less phenotype.

The induced mutations in CsbHLH genes of 3 lines $(4,15$, 29) from the spare root hair group and 4 lines $(2,6,9,10)$ from the root hair-less group were further characterized via sequencing targeted amplicons (Figures 7C,D). For the CsbHLH82 gene, induced mutations occurred in all tested hairy root lines (Figure 7C). Various types of indels were observed at the target locations, in which the deletion sizes ranged from -1 bp up to $-33 \mathrm{bp}$ and the insertions were from +1 to $+10 \mathrm{bp}$. Different from CsbHLH82, targeted mutations in CsbHLH66 were confirmed in only 5 of 7 tested root hair lines, including 2 mutant alleles in lines 2 and 15, which were undetectable in heteroduplex analysis because of only $1 \mathrm{bp}$ indels (1 bp insertion or deletion). The $1 \mathrm{bp}$ insertion and big deletions ( -16 to $-49 \mathrm{bp}$ ) in the CsbHLH66 targeted gene were found in 3 tested hair-less root lines, while $1 \mathrm{bp}$ deletion was found in one sparse root hair line 15 only (Figure 7D). Moreover, two mutant alleles (biallelic mutation) of targeted genes were also observed in many hairy root lines such as lines 6 and 10 for CsbHLH66 gene and lines 2, 6, 15, and 29 of CsbHLH82 gene (Figures 7C,D). The homozygous mutant allele was found in line 9 (for CsbHLH82 gene). Three different alleles (chimeric alleles) of the CsbHLH82 gene were also observed in hairy root line 4 . Importantly, lines 6 and 10 contained null mutations of both the CsbHLH82 and CsbHLH66 genes. As a consequence, it is obvious that our developed hairy root system has a great potential for genome editing studies in cucumber.

The stability of induced mutations of targeted genes was further confirmed in in vitro propagated hairy roots. The root fragments from line 6 and 10 were cut and propagated in a new medium for the next generations. Heteroduplex, Sanger, and amplicon sequencing analysis were conducted using DNA 
A

Target 1

CGTCGGCGATACAACTCCGG

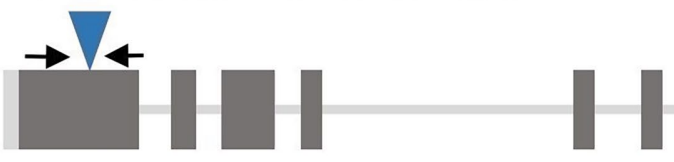

CsbHLH66

Target 2

$1 \mathrm{~kb}$

GGGGAACCCTGGAATTGGGGTAG

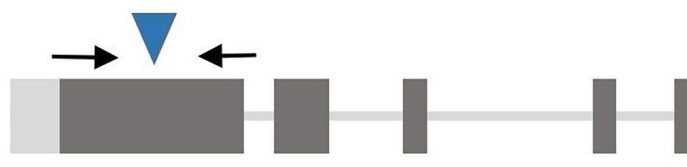

CsbHLH82

$1 \mathrm{~kb}$

B

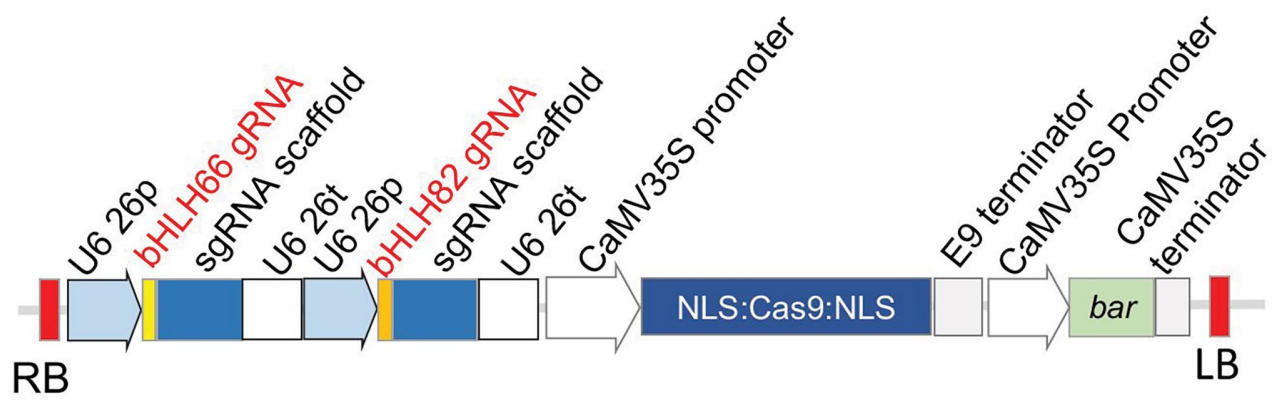

C
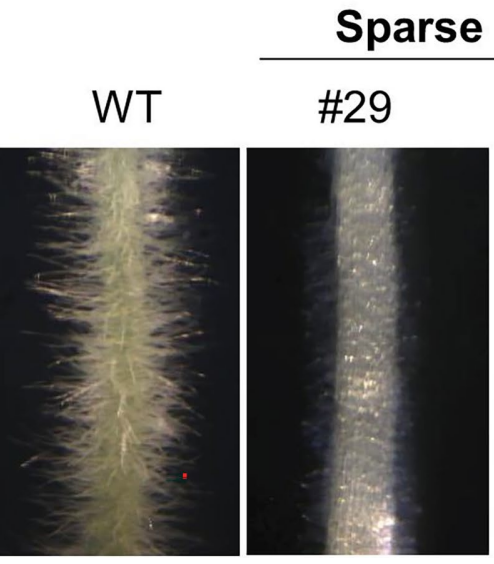

root hair \#4

Root hair-less
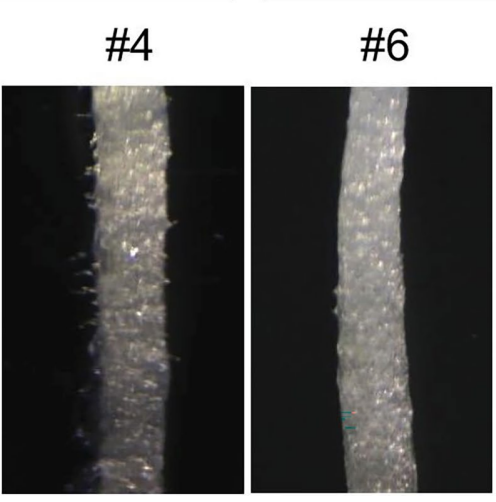

\#10

FIGURE 6 | CRISPR/Cas9 construction and phenotyping of mutant hairy roots. (A) Maps of CsbHLH66, CsbHLH82 genes and target locations. The black arrows indicate primer binding sites for genotyping. (B) T-DNA regions of the CRISPR/Cas9 plant transformation vector. (C) Root hair phenotypes of WT and mutant hairy roots. The red scale bar indicates $1 \mathrm{~mm}$.

extracted from the third propagation root lines. DNA band patterns in PAGE and sequencing data indicated that the mutations observed in the propagated lines were the same as in the primary roots (first propagation) for both CsbHLH82 and CsbHLH66 (Supplementary Figure S4). This result demonstrated that stably induced mutations were conducted in cucumber hairy roots.

\section{DISCUSSION}

\section{Establishment of an Efficient Procedure for Cucumber in vitro Hairy Root Induction}

In this study, an efficient procedure for cucumber in vitro hairy root induction was established. The hairy root induction frequency could reach $100 \%$ after 20 days of co-cultivation 
A

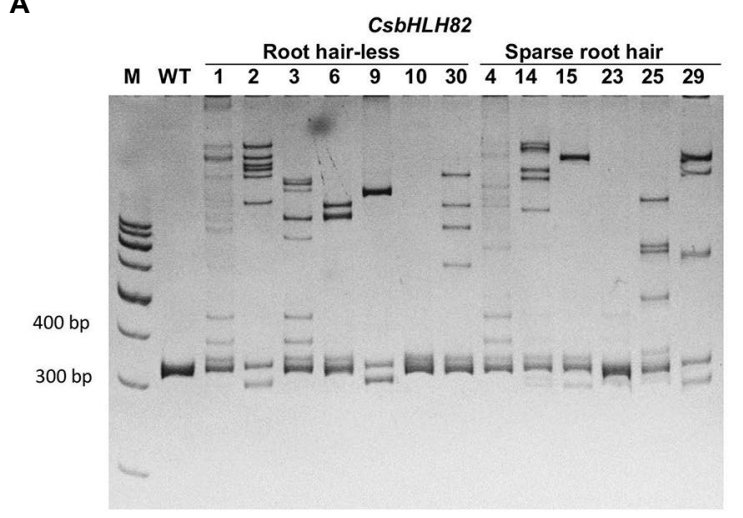

B

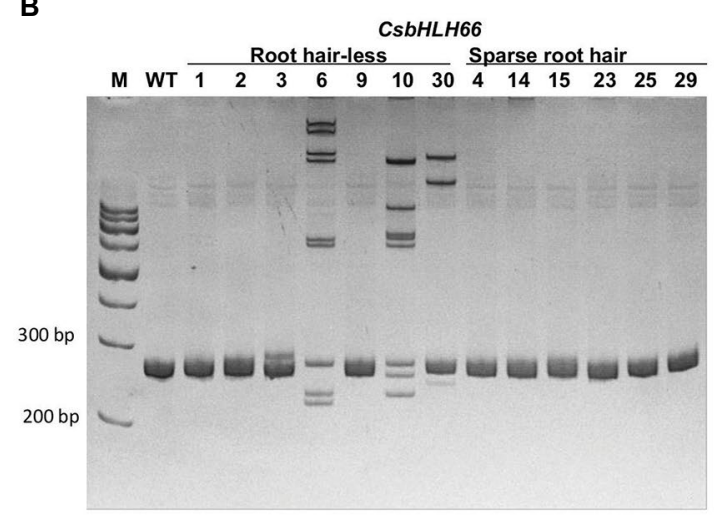

C

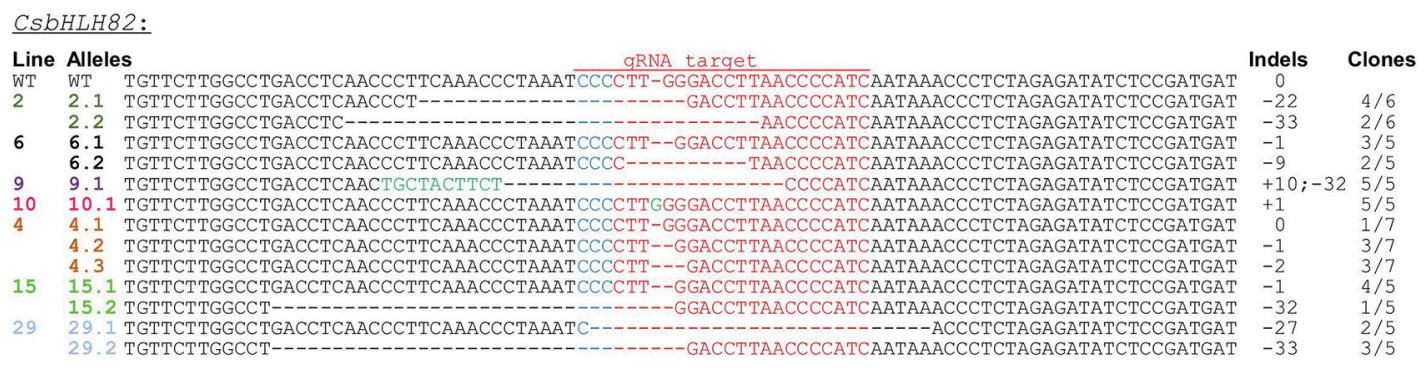

D

CSBHLH66:

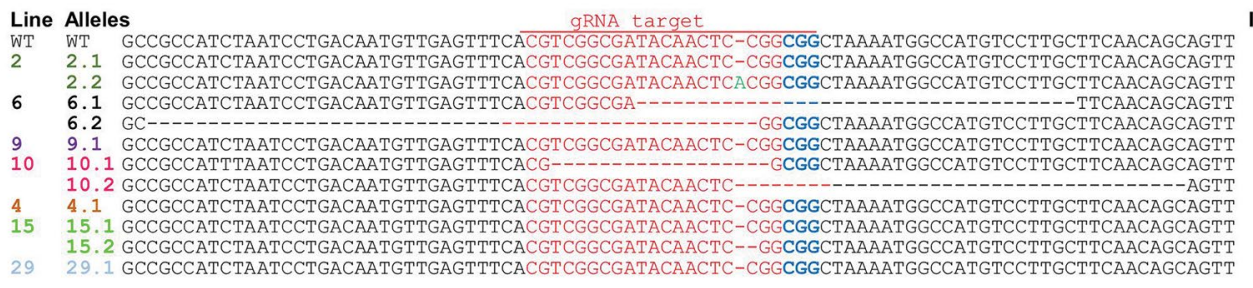

$\begin{array}{ccc} & \text { Indels } & \text { Clones } \\ \mathrm{T} & 0 & \\ \mathrm{~T} & 0 & 2 / 5 \\ \mathrm{~T} & +1 & 3 / 5 \\ \mathrm{~T} & -35 & 1 / 5 \\ \mathrm{~T} & -49 & 4 / 5 \\ \mathrm{~T} & 0 & 5 / 5 \\ \mathrm{~T} & -16 & 1 / 6 \\ \mathrm{~T} & -35 & 5 / 6 \\ \mathrm{~T} & 0 & 5 / 5 \\ \mathrm{~T} & 0 & 2 / 5 \\ \mathrm{~T} & -1 & 3 / 5 \\ \mathrm{~T} & 0 & 5 / 5\end{array}$

FIGURE 7 | Identification and characterization of induced mutations in cucumber hairy roots. (A,B) Heteroduplex analysis of CsbHLH82 and CsbHLH66, respectively. M: 100 bp DNA Ladder; WT: wild type. (C,D) Expanding targeted sequences of CsbHLH82 and CsbHLH66 genes. Red letters indicate target sequences. Blue letters refer to PAM sequences. Green letters indicate inserted nucleotides. The indels indicate targeted sequence changes: 0 for no change, - for deletion, + for insertion. The number of clones used for sequencing was indicated.

using the R. rhizogenes K599 strain. Different factors including explants, co-inoculation, and co-cultivation conditions have been reported to influence plant transformation efficacy as well as hairy root induction. In our results, the 5 days co-cultivation was selected because of the highest hairy root induction efficiency. Co-cultivation duration extended to 7 days negatively influenced cucumber hairy root formation due to the overgrowth of bacteria after inoculation. This result is in agreement with Shi et al. (2006), in which 5 days of co-cultivation was considered to be optimal for cucumber in vitro hairy root induction. One other optimization in this study was the use of cotyledon pieces from 2-day-old seedlings as initial explants instead of 10-day-old seedlings as in previous studies (Shi et al., 2006; Shi and Lindemann, 2006) for hairy root generation. Such kind of explants was reported to be optimal for cucumber transformation and regeneration only (Nanasato et al., 2013). Apart from increasing the number of hair roots produced, the use of younger cotyledons also helps to shorten the time needed for hairy root induction procedure ( $<1$ month).

\section{Rhizobium rhizogenes K599 Strain Is Effective for Cucumber Hairy Root Induction}

The hairy roots were effectively induced from the local cultivar Choka F1 using $R$. rhizogenes K599 strain. Bacterial strains have been known as an important factor for hairy root induction and transformation. In previous studies, different $R$. rhizogenes strains were used for in vitro hairy root induction in cucumber (Trulson et al., 1986; Shi et al., 2006; Shi and Lindemann, 2006; Anuar et al., 2011; JingLong et al., 2017) and the hairy root induction rates were variable depending on cucumber cultivars and transformation procedures. The hairy root induction rate of over 90\% was reported by Shi et al. (2006) and Shi and Lindemann (2006) using the ATCC 15834 strain. Recently, 
R. rhizogenes $\mathrm{K} 599$ strain had been utilized for inducing hairy roots in cucumber with the frequency of $95 \%$ under in vivo conditions (Fan et al., 2020). In our study, the K599 strain could facilitate $100 \%$ infected cucumber explants to produce strong hairy roots, much higher than that of ATCC 15834 strain $(83.3 \pm 16.6 \%)$. The formation of callus instated of hairy roots by ATCC 15834 strain (Supplementary Figure S1) might be a reason to lower the number of hairy roots produced. The fewer callus formed from K599 inoculation were coupled with the better hairy root morphology. Therefore, the K599 strain is most suitable for hairy root induction and transformation of the local cultivar.

\section{A Convenient Tool to Assess the Activities of CRISPR/Cas9 Constructs in Cucumber}

Hairy root systems have been used for genome editing of various plant species. For cucumber, the hairy root system has been applied for studying transgenic plant generation (Trulson et al., 1986), gene expression (Fan et al., 2020), promoter evaluation (Anuar et al., 2011), recombinant protein production (Shi and Lindemann, 2006), but not for genome editing yet. In this study, an efficient procedure for in vitro hairy root induction of a local cucumber cultivar (ChokaF1), which was successfully utilized for stable transformation (Hoang et al., 2021). This procedure was well performed for the expression of a reporter gene $(g u s)$ in a short time. The developed hairy root system was also effective for gene editing of cucumber using CRISPR/Cas9 construct. Targeted mutations of two endogenous genes ( $\mathrm{CsbHLH66}$ and CsbHLH82) were induced accurately. Different indels of targeted genes were observed in tested hair roots including insertions, and larger and small deletions (Figure 7). All homozygous, heterozygous, and biallelic, as well as chimeric alleles could be obtained in the primary hairy roots (Figure 7). In addition, the targeting capability of different sgRNAs (single guide RNA) could be evaluated within 1 month, instated of several months or longer if using the transgenic plant approach. The targeted mutations induced in transgenic hairy roots were also observed in stable transgenic cucumbers (Chandrasekaran et al., 2016; Hu et al., 2017).

\section{CsbHLH66 and CsbHLH82 Genes Are Involved in Root Hair Performance of Cucumber}

The root hair-less phenotype was observed in Lotus japonicus and characterized as a consequence of natural mutations in the SjRHL1 gene (Karas et al., 2009). In A. thaliana, this phenotype was also found in the triple mutant of AtLRL1, $A t L R L 2$, and AtLRL3, while sparse root hair phenotypes were caused by single or double mutations of these three genes (Karas et al., 2009). In our study, induced mutations in CsbHLH82 resulted in the root hair spare phenotype (lines 15, 29), while simultaneous mutations of both CsbHLH82 and CsbHLH66 genes caused the root hair-less phenotype (lines 6, 10; Figures 7C,D). This result suggests that two bHLH transcription factors encoded by CsbHLH82 and CsbHLH66 are redundantly involved in regulating the root hair formation in cucumber.
However, further research needs to be performed to characterize and confirm the contributions of CsbHLH66, CsbHLH82, and other $C s b H L H$ genes in cucumber root hair formation at whole plant levels.

\section{CONCLUSION}

In this study, an effective protocol for cucumber hairy root induction and transformation was developed for a local cucumber cultivar. This hairy root system has been successfully used to evaluate the expression of transgenes and the activities of CRISPR/Cas9 systems within 1 month. The obtained result obviously provides a potential tool for transgene expression and genome editing studies in cucumber as well as other Cucumis plants.

\section{DATA AVAILABILITY STATEMENT}

The original contributions presented in the study are included in the article/Supplementary Material, further inquiries can be directed to the corresponding authors.

\section{AUTHOR CONTRIBUTIONS}

$\mathrm{PD}$ and $\mathrm{HC}$ conceived and supervised the study. PD, DN, and $\mathrm{TH}$ designed the experiments, analyzed the data and prepared the manuscript. CN conducted the phylogenetic tree and designed the gRNAs. NL and HT performed CRISPR/ Cas9 vector constructions. TH conducted cucumber hairy root transformation. DN performed genotyping and phenotyping of transgenic hairy roots. $\mathrm{HC}, \mathrm{Y}-\mathrm{HM}$, and $\mathrm{CN}$ revised and proofread the manuscript. All authors contributed to the article and approved the submitted version.

\section{FUNDING}

This research was supported by the National Foundation for Science and Technology of Vietnam (106.03-2019.11) and the Vietnam Academy of Science and Technology (ĐLTE00.10/20-21).

\section{ACKNOWLEDGMENTS}

The authors thank Van Tuong Nguyen for proofreading of the manuscript and helpful suggestions.

\section{SUPPLEMENTARY MATERIAL}

The Supplementary Material for this article can be found online at: https://www.frontiersin.org/articles/10.3389/fpls.2021.770062/ full\#supplementary-material 


\section{Supplementary Table S1 | Primers used in this study.}

Supplementary Figure S1 | Cucumber hairy root induction by Rhizobium rhizogenes strain K599 and ATCC 15834. The photos were captured at 10 days after co-cultivation.

Supplementary Figure S2 | GUS histochemical analysis of the herbicide resistant hairy roots. WT: non-transgenic hairy roots grown on the PPT free medium; 1-14: Hairy root lines survived and developed on the selection medium with PPT.

Supplementary Figure S3 | Distribution of root hair phenotypes of tested cucumber hairy roots.

\section{REFERENCES}

Anuar, M., Ismail, I., and Zainal, Z. (2011). Expression analysis of the $35 \mathrm{~S}$ $C a M V$ promoter and its derivatives in transgenic hairy root cultures of cucumber (Cucumis sativus) generated by Agrobacterium rhizogenes infection. Afr. J. Biotechnol. 10, 8236-8244. doi: 10.5897/AJB11.130

Bueno dos Reis, L., Lemes da Silva, M., Barcelos Passos Lima, A., Peixoto de Oliveira, M. L., Lopes Paim Pinto, D., Ribeiro Garcia Lani, E., et al. (2007). Agrobacterium rhizogenes-mediated transformation of passionfruit species: Passiflora cincinnata and P. edulis f. flavicarpa. Acta Hortic. 738, 425-431. doi: 10.17660/ActaHortic.2007.738.51

Butler, N. M., Jansky, S. H., and Jiang, J. (2020). First-generation genome editing in potato using hairy root transformation. Plant Biotechnol. J. 18, 2201-2209. doi: 10.1111/pbi.13376

Chandrasekaran, J., Brumin, M., Wolf, D., Leibman, D., Klap, C., Pearlsman, M., et al. (2016). Development of broad virus resistance in non-transgenic cucumber using CRISPR/Cas9 technology. Mol. Plant Pathol. 17, 1140-1153. doi: $10.1111 / \mathrm{mpp} .12375$

Chee, P. P. (2001). “Transgenic cucumber (Cucumis sativus L.)," in Transgenic Crops II. ed. Y. P. S. Bajaj (Berlin, Heidelberg: Springer Berlin Heidelberg), 132-146.

Chen, J., Zhang, X., Wang, T., Li, Z., Guan, G., and Hong, Y. (2012). Efficient detection, quantification and enrichment of subtle allelic alterations. DNA Res. 19, 423-433. doi: 10.1093/dnares/dss023

Chojak-Koźniewska, J., Kuźniak, E., and Zimny, J. (2018). The effects of combined abiotic and pathogen stress in plants: insights From salinity and Pseudomonas syringae pv lachrymans interaction in cucumber. Front. Plant Sci. 9:1691. doi: $10.3389 /$ fpls.2018.01691

Doyle, J. J., and Doyle, J. L. (1987). A rapid DNA isolation procedure for small quantities of fresh leaf tissue. Phytochem. Bull. 19, 11-15.

Du, H., Zeng, X., Zhao, M., Cui, X., Wang, Q., Yang, H., et al. (2016). Efficient targeted mutagenesis in soybean by TALENs and CRISPR/Cas9. J. Biotechnol. 217, 90-97. doi: 10.1016/j.jbiotec.2015.11.005

Elsheery, N. I., Helaly, M. N., Omar, S. A., John, S. V. S., Zabochnicka-Swiątek, M., Kalaji, H. M., et al. (2020). Physiological and molecular mechanisms of salinity tolerance in grafted cucumber. S. Afr. J. Bot. 130, 90-102. doi: 10.1016/j.sajb.2019.12.014

Fan, Y., Xu, F., Zhou, H., Liu, X., Yang, X., Weng, K., et al. (2020). A fast, simple, high efficient and one-step generation of composite cucumber plants with transgenic roots by Agrobacterium rhizogenes-mediated transformation. Plant Cell Tissue Organ Cult. 141, 207-216. doi: 10.1007/s11240-020-01781-x

Gutierrez-Valdes, N., Häkkinen, S. T., Lemasson, C., Guillet, M., Oksman-Caldentey, K.-M., Ritala, A., et al. (2020). Hairy root cultures-a versatile tool with multiple applications. Front. Plant Sci. 11:33. doi: 10.3389/ fpls.2020.00033

Hoang, T. H. T., Hoang, L. K., Nguyen, L. T., Trinh, D. D., Le, T. N. T., Pham, N. B., et al. (2021). Study in vitro regeneration and transformation systems of cucumber variety Choka F1 using gus reporter gene. TNU J. Sci. Technol. 226, 83-91. (Vietnamese with an English abstract)

Hu, B., Li, D., Liu, X., Qi, J., Gao, D., Zhao, S., et al. (2017). Engineering non-transgenic gynoecious cucumber using an improved transformation protocol and optimized CRISPR/Cas9 system. Mol. Plant 10, 1575-1578. doi: 10.1016/j.molp.2017.09.005

Jacobs, T. B., and Martin, G. B. (2016). High-throughput CRISPR vector construction and characterization of DNA modifications by generation of tomato hairy roots. J. Vis. Exp. 110:53843. doi: 10.3791/53843
Supplementary Figure S4 | The stability of induced mutations in propagated cucumber hairy roots. (A) Heteroduplex analysis of $\mathrm{CsbHLH66}$ gene. (B) Heteroduplex analysis of CsbHLH82 gene. M: 100 bp DNA Ladder; WT: wild type; P6 and P10: The original hairy roots of lines 6 and 10; 6-1, 6-1-1, 10-1, 10-1-1: Propagated roots from lines 6 and 10 at the second and third generations. (C,D) Expanding targeted sequences of CsbHLH82 and CsbHLH66 genes from propagated roots of the hairy root lines 6 and 10. Amplicon sequencings were conducted by the Sanger method. The expected sequences of mutant alleles were denoted upper chromatograms. (E) Clone sequencing of $\mathrm{Cs} b \mathrm{HLH} 82$ and $\mathrm{Cs} b H \mathrm{HLH} 66$ targeted regions of propagated cucumber hairy roots at the third generation. Red and blue letters indicate target and PAM sequences, respectively. Green letters indicate inserted nucleotides.

Jefferson, R. A., Kavanagh, T. A., and Bevan, M. W. (1987). GUS fusions: beta-glucuronidase as a sensitive and versatile gene fusion marker in higher plants. EMBO J. 6, 3901-3907. doi: 10.1002/j.1460-2075.1987. tb02730.x

JingLong, J., Chao, J., Li, L., JiXue, S., Yun, T., and XuMing, R. (2017). Studies on effects of different conditions on induction rate of hairy roots in Cucumis sativus L. J. Agric. Sci. Technol. 19, 24-30. doi: 10.13304/j.nykjdb.2016. 581

Karas, B., Amyot, L., Johansen, C., Sato, S., Tabata, S., Kawaguchi, M., et al. (2009). Conservation of lotus and Arabidopsis basic helix-loop-helix proteins reveals new players in root hair development. Plant Physiol. 151, 1175-1185. doi: $10.1104 /$ pp.109.143867

Karas, B., Murray, J., Gorzelak, M., Smith, A., Sato, S., Tabata, S., et al. (2005). Invasion of Lotus japonicus root hairless 1 by Mesorhizobium loti involves the nodulation factor-dependent induction of root hairs. Plant Physiol. 137, 1331-1344. doi: 10.1104/pp.104.057513

Kumar, S., Stecher, G., Li, M., Knyaz, C., and Tamura, K. (2018). MEGA X: molecular evolutionary genetics analysis across computing platforms. Mol. Biol. Evol. 35, 1547-1549. doi: 10.1093/molbev/msy096

Le, H., Nguyen, N. H., Ta, D. T., Le, T. N. T., Bui, T. P., Le, N. T., et al. (2020). CRISPR/Cas9-mediated knockout of Galactinol synthase-encoding genes reduces raffinose family oligosaccharide levels in soybean seeds. Front. Plant Sci. 11:612942. doi: 10.3389/fpls.2020.612942

Li, C., Nguyen, V., Liu, J., Fu, W., Chen, C., Yu, K., et al. (2019). Mutagenesis of seed storage protein genes in soybean using CRISPR/Cas9. BMC Res. Notes 12:176. doi: 10.1186/s13104-019-4207-2

Liu, L., Duan, L., Zhang, J., Mi, G., Zhang, X., Zhang, Z., et al. (2013). Arabidopsis LOS5 gene enhances chilling and salt stress tolerance in cucumber. J. Integr. Agric. 12, 825-834. doi: 10.1016/S2095-3119(13)60270-1

Michno, J.-M., Wang, X., Liu, J., Curtin, S. J., Kono, T. J., and Stupar, R. M. (2015). CRISPR/Cas mutagenesis of soybean and Medicago truncatula using a new web-tool and a modified Cas9 enzyme. GM Crops Food 6, 243-252. doi: 10.1080/21645698.2015.1106063

Murashige, T., and Skoog, F. (1962). A revised medium for rapid growth and bio assays with tobacco tissue cultures. Physiol. Plant. 15, 473-497. doi 10.1111/j.1399-3054.1962.tb08052.x

Nanasato, Y., Konagaya, K.-I., Okuzaki, A., Tsuda, M., and Tabei, Y. (2013). Improvement of Agrobacterium-mediated transformation of cucumber (Cucumis sativus L.) by combination of vacuum infiltration and co-cultivation on filter paper wicks. Plant Biotechnol. Rep. 7, 267-276. doi: 10.1007/ s11816-012-0260-1

Paris, H. S., Daunay, M.-C., and Janick, J. (2012). Occidental diffusion of cucumber (Cucumis sativus) 500-1300 CE: two routes to Europe. Ann. Bot. 109, 117-126. doi: 10.1093/aob/mcr281

Plader, W., Burza, W., and Malepszy, S. (2007). "Cucumber," in Transgenic Crops IV. eds. E.-C. Pua and M. R. Davey (Berlin, Heidelberg: Springer Berlin Heidelberg), 181-199.

Shi, H.-P., and Lindemann, P. (2006). Expression of recombinant Digitalis lanata EHRH. Cardenolide 16'-O-glucohydrolase in Cucumis sativus L. hairy roots. Plant Cell Rep. 25, 1193-1198. doi: 10.1007/s00299-006-0183-9

Shi, H.-P., Qi, Y., Zhang, Y., and Liang, S. (2006). Induction of cucumber hairy roots and effect of cytokinin 6-BA on its growth and morphology. Sheng Wu Gong Cheng Xue Bao 22, 514-520.

Stemmer, M., Thumberger, T., Del Sol Keyer, M., Wittbrodt, J., and Mateo, J. L. (2015). CCTop: an intuitive, flexible and reliable CRISPR/Cas9 target 
prediction tool. PLoS One 10:e0124633. doi: 10.1371/journal.pone. 0124633

Taha, N., Abdalla, N., Bayoumi, Y., and El-Ramady, H. (2020). Management of greenhouse cucumber production under arid environments: a review. Environ. Biodivers. Soil Secur. 4. doi: 10.21608/JENVBS.2020.30729. 1097

Trulson, A. J., Simpson, R. B., and Shahin, E. A. (1986). Transformation of cucumber (Cucumis sativus L.) plants with Agrobacterium rhizogenes. Theor. Appl. Genet. 73, 11-15. doi: 10.1007/BF00273711

Xing, H.-L., Dong, L., Wang, Z.-P., Zhang, H.-Y., Han, C.-Y., Liu, B., et al. (2014). A CRISPR/Cas9 toolkit for multiplex genome editing in plants. BMC Plant Biol. 14:327. doi: 10.1186/s12870-014-0327-y

Zeng, P., Vadnais, D. A., Zhang, Z., and Polacco, J. C. (2004). Refined glufosinate selection in Agrobacterium-mediated transformation of soybean [Glycine $\max$ (L.) Merrill]. Plant Cell Rep. 22, 478-482. doi: 10.1007/ s00299-003-0712-8

Zhang, H., Cao, Y., Zhang, H., Xu, Y., Zhou, C., Liu, W., et al. (2020). Efficient generation of CRISPR/Cas9-mediated homozygous/biallelic Medicago truncatula mutants using a hairy root system. Front. Plant Sci. 11:294. doi: 10.3389/ fpls.2020.00294
Zhang, D., Zhang, Z., Unver, T., and Zhang, B. (2021). CRISPR/Cas: a powerful tool for gene function study and crop improvement. J. Adv. Res. 29, 207-221. doi: $10.1016 /$ j.jare.2020.10.003

Conflict of Interest: The authors declare that the research was conducted in the absence of any commercial or financial relationships that could be construed as a potential conflict of interest.

Publisher's Note: All claims expressed in this article are solely those of the authors and do not necessarily represent those of their affiliated organizations, or those of the publisher, the editors and the reviewers. Any product that may be evaluated in this article, or claim that may be made by its manufacturer, is not guaranteed or endorsed by the publisher.

Copyright (๑) 2022 Nguyen, Hoang, Le, Tran, Nguyen, Moon, Chu and Do. This is an open-access article distributed under the terms of the Creative Commons Attribution License (CC BY). The use, distribution or reproduction in other forums is permitted, provided the original author(s) and the copyright owner(s) are credited and that the original publication in this journal is cited, in accordance with accepted academic practice. No use, distribution or reproduction is permitted which does not comply with these terms. 